\begin{tabular}{|c|l|}
\hline Title & Coherent manipulation of nuclear spins using spin injection from a half-metallic spin source \\
\hline Author(s) & Uemura, Tetsuy a; A kiho, Takafumi; Ebina, Y uy a; Y amamoto, Masafumi \\
\hline Citation & $\begin{array}{l}\text { Physical review B, 91(14), 140410 } \\
\text { https://doi.org/10.1103/PhysRevB.91.140410 }\end{array}$ \\
\hline Issue Date & 2015-04 29 \\
\hline Doc URL & http://hdl.handle.net/2115/59184 \\
\hline Rights & @2015A merican Physical Society \\
\hline Type & article \\
\hline File Information & PhysRevB.91.140410.pdf \\
\hline
\end{tabular}

Instructions for use 


\title{
Coherent manipulation of nuclear spins using spin injection from a half-metallic spin source
}

\author{
Tetsuya Uemura," Takafumi Akiho, Yuya Ebina, and Masafumi Yamamoto \\ Division of Electronics for Informatics, Graduate School of Information Science and Technology, \\ Hokkaido University, Sapporo 060-0814, Japan
}

(Received 29 December 2014; revised manuscript received 13 April 2015; published 29 April 2015)

\begin{abstract}
We have developed a nuclear magnetic resonance (NMR) system that uses spin injection from a highly polarized spin source. Efficient spin injection into GaAs from a half-metallic spin source of $\mathrm{Mn}$-rich $\mathrm{Co}_{2} \mathrm{MnSi}_{\mathrm{S}}$ enabled an efficient dynamic nuclear polarization of Ga and As nuclei in GaAs and a sensitive detection of NMR signals. Moreover, coherent control of nuclear spins, or the Rabi oscillation between two quantum levels formed at Ga nuclei, induced by a pulsed NMR has been demonstrated at a relatively low magnetic field of $\sim 0.1 \mathrm{~T}$. This provides a novel all-electrical solid-state NMR system with the high spatial resolution and high sensitivity needed to implement scalable nuclear-spin-based qubits.
\end{abstract}

DOI: 10.1103/PhysRevB.91.140410

PACS number(s): 72.25.Dc, 72.25.Hg, 76.60.-k, 76.70.Fz

Nuclear spins in semiconductors are an ideal system for implementing quantum bits (qubits) for quantum computation because they have an extremely long coherence time. The nuclear magnetic resonance (NMR) technique enables the control and detection of nuclear-spin qubits, and quantum algorism with seven qubits has been demonstrated in molecules in a liquid [1]. For large-scale integration, however, the implementation of qubits in solid-state materials, especially in semiconductors, is indispensable. Moreover, in conventional NMR techniques, a strong magnetostatic field should be applied to polarize nuclear spins, making it difficult to selectively control nuclear spins located within nanometer-sized regions. Furthermore, since the magnetic moment of a nuclear spin is three orders of magnitude smaller than that of an electron spin, the sensitivity of detecting nuclear spins through a pickup coil is quite low. Thus, there is a strong need to develop a novel NMR technique with high spatial resolution and high sensitivity to enable a future large-scale quantum computing system. From this point of view, dynamic nuclear polarization (DNP), where nuclear spins are dynamically polarized through a hyperfine interaction between nuclear spins and electron spins, has attracted much interest, since it can drastically increase the NMR signal. Several solid-state NMR devices based on different DNP techniques by optical [2-4] or electrical means [5-7] have recently been reported. Furthermore, coherent manipulation of nuclear spins, or the Rabi oscillation, which is a key factor for nuclear-spin-based qubits, has been demonstrated electrically in GaAs/AlGaAs quantum Hall systems [8-10] and optically in GaAs/AlGaAs quantum wells [11,12]. Although the optical method is suitable for clarifying the fundamental physics relevant to nuclear spins, it is restricted in its scalability because the spatial resolution is limited by the optical wavelength. Quantum Hall systems, on the other hand, require a relatively strong magnetic field of several tesla and a low temperature below $1 \mathrm{~K}$ to create the highly spin-polarized electrons necessary for the DNP and the detection of nuclear-spin states.

An injection of spin-polarized electrons from a ferromagnetic electrode into a semiconductor also creates spinpolarized electronic states in a nanoscale semiconductor region

\footnotetext{
*uemura@ist.hokudai.ac.jp
}

electrically, thus enabling a nanoscale DNP system without a strong magnetic field. There have been several reports on spin injection into GaAs [13-18], Si [19-21], and Ge [22], and on NMR along with DNP using spin injection into GaAs [23-26]. However, all of the work on NMR based on spin injection has been limited to static investigation, because the spin injection efficiency in most of these devices is on the order of a few percent. A half-metallic ferromagnet could be considered an excellent candidate for a spin source for efficient DNP because it provides complete spin polarization at the Fermi level. In previous studies, we demonstrated high tunneling magnetoresistance (TMR) ratios of up to $1995 \%$ at $4.2 \mathrm{~K}$ and up to $354 \%$ at $290 \mathrm{~K}$ in magnetic tunnel junctions (MTJs) having $\mathrm{Mn}$-rich $\mathrm{Co}_{2} \mathrm{MnSi}$ (CMS) electrodes [27], and 2610\% at $4.2 \mathrm{~K}$ and $429 \%$ at $290 \mathrm{~K}$ in $\mathrm{Co}_{2}(\mathrm{Mn}, \mathrm{Fe}) \mathrm{Si}$ (CMFS) MTJs [28]. We have experimentally shown that harmful defects in CMS, CMFS, and $\mathrm{Co}_{2} \mathrm{MnGe}$ thin films-i.e., $\mathrm{Co}_{\mathrm{Mn}}$ antisites - can be suppressed by a Mn-rich composition [27-32]. Furthermore, we have achieved efficient spin injection from Mn-rich CMS into GaAs via an ultrathin $\mathrm{Co}_{50} \mathrm{Fe}_{50}(\mathrm{CoFe})$ insertion layer, resulting in electron spin polarization $\left(P_{\mathrm{GaAs}}\right)$ of up to $52 \%$ at ferromagnet/GaAs interface at $4.2 \mathrm{~K}$ [33]. This value is more than one order of magnitude higher than those obtained for a Fe electrode or a $\mathrm{CoFe}$ electrode [16]. $P_{\mathrm{GaAs}}$ of $27 \%$ and $51 \%$ has also been reported in spin light-emitting diodes with $\mathrm{Co}_{2} \mathrm{MnGe}$ and $\mathrm{Co}_{2} \mathrm{FeSi}$ electrodes, respectively $[34,35]$. We also demonstrated that the high spin polarization of Mn-rich CMS electrodes makes possible efficient DNP in GaAs, and we clarified the transient response of nuclear spins against a change in the applied magnetic field through a transient oblique Hanle effect measurement [36]. In this Rapid Communication we report coherent manipulation of nuclear spins through spin injection from a half-metallic spin source of Mn-rich CMS. This opens a new route to an all-electrical solid-state NMR system that can be used to implement scalable nuclear-spin-based qubits.

Figures 1(a) and 1(b) show a schematic structure and a layer structure for the solid-state NMR device investigated in this study. The nuclear spins in a semiconductor channel are initialized through a DNP technique using spin-polarized electrons injected from contact-2. The nuclear-spin states are then manipulated through the NMR by irradiation of a radiofrequency (rf) magnetic field. The manipulated nuclear-spin 


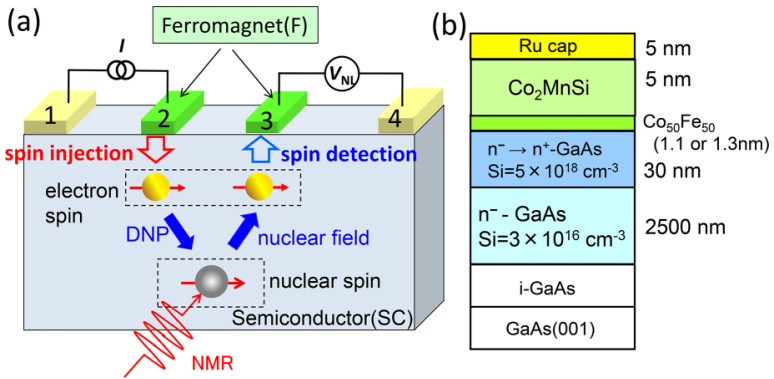

FIG. 1. (Color online) (a) Schematic structure of an all-electrical solid-state NMR system using spin injection. (b) Layer structure of the spin injection device fabricated in this study.

states can be read out through detection of the nonlocal voltage $\left(V_{\mathrm{NL}}\right)$ between contact-3 and contact-4 since the nuclear magnetic field produced by nuclear-spin polarization induces the Larmor precession of electron spins below detector contact-3.

The layer structure consists of 2.5- $\mu$ m-thick $n^{-}$-GaAs with a doping concentration of $3 \times 10^{16} \mathrm{~cm}^{-3}$ as a spin-transport channel and a $\operatorname{CMS}(5 \mathrm{~nm}) / \mathrm{CoFe}(1.1$ or $1.3 \mathrm{~nm})$ bilayer as a spin source. A Mn-rich CMS film with a Mn composition of 1.30 in the expression of $\mathrm{Co}_{2} \mathrm{Mn}_{1.30} \mathrm{Si}_{\beta} \quad(\beta=0.88$ or 0.84) was chosen to suppress the harmful $\mathrm{Co}_{\mathrm{Mn}}$ antisites to improve the half-metallicity. A 15 -nm-thick $n^{-} \rightarrow n^{+}$-GaAs transition layer and a 15-nm-thick $n^{+}$-GaAs layer with a doping concentration of $5 \times 10^{18} \mathrm{~cm}^{-3}$ were inserted between the channel and ferromagnetic electrodes to form a narrow Schottky barrier. Using electron beam lithography and $\mathrm{Ar}$ ion milling techniques, lateral spin-transport devices as shown in Fig. 1(a) were fabricated. The size of the injector contact (contact-2) and detector contact (contact-3) were $0.5 \times 10 \mu \mathrm{m}$ and $1.0 \times 10 \mu \mathrm{m}$, respectively, and the spacing between the contacts was $0.5 \mu \mathrm{m}$. The detailed fabrication method of the layer structure and a four-terminal lateral spin-transport device is described elsewhere [33,36]. The creation, control, and detection of nuclear-spin states in GaAs were evaluated in a four-terminal nonlocal geometry where $V_{\mathrm{NL}}$ between contact- 3 and contact- 4 was measured under a constant current (I) supplied between contact- 2 and contact-1 under a static magnetic field (B) and an rf magnetic field ( $\left.\mathbf{B}_{\mathbf{a c}}\right)$. The $\mathbf{B}_{\mathbf{a c}}$ was generated by an 11-turn coil with a diameter of $1.0 \mathrm{~cm}$ for the NMR experiment. All the measurements were done at $4.2 \mathrm{~K}$.

We observed clear spin-valve signals and Hanle signals (not shown) in fabricated devices with a CMS spin source, providing direct evidence of spin injection, detection, and transport in GaAs $[33,36]$. The spin lifetime $\left(\tau_{s}\right)$ and spin diffusion length $\left(l_{\mathrm{sf}}\right)$ in the $n$-GaAs channel with a $\mathrm{CMS} / \mathrm{CoFe}(1.1$ or $1.3 \mathrm{~nm}$ ) spin source, estimated from Hanle signals, were typically $20 \mathrm{~ns}$ and $3 \mu \mathrm{m}$, respectively. We now describe the electrical detection of continuous-wave (cw) NMR using a transient oblique Hanle effect measurement. When nuclear spins are polarized by the DNP, the electron spins are affected by the nuclear field, or the Overhauser field $\left(\mathbf{B}_{\mathbf{n}}\right)$, through the hyperfine interactions. The time evolution of the nuclear field can be described by [23]

$$
\mathbf{B}_{\mathbf{n}}(t)=\left[\mathbf{B}_{\mathbf{n}}(0)-\mathbf{B}_{\mathbf{n}}(\infty)\right] \exp \left(-t / t_{c}\right)+\mathbf{B}_{\mathbf{n}}(\infty),
$$

where $t_{c}$ is the characteristic time needed for nuclear spins to reach a steady state, and $\mathbf{B}_{\mathbf{n}}(\infty)$ is a steady-state Overhauser field, which is given by [37]

$$
\mathbf{B}_{\mathbf{n}}(\infty)=f_{1} b_{n} \frac{\mathbf{B} \cdot \mathbf{S}}{\mathbf{B}^{2}+\xi B_{l}^{2}} \mathbf{B},
$$

where $f_{1}(\leqslant 1)$ is the leakage factor, $b_{n}$ is the effective field due to the polarization of nuclear spins, which takes the negative value of $-17 \mathrm{~T}$ in GaAs for the theoretical ideal case, $\mathbf{S}$ is the average electron spin $(|\mathbf{S}|=1 / 2$ corresponds to $P_{\mathrm{GaAs}}=100 \%$ ), $\mathbf{B}$ is the external magnetic field, $B_{1}$ is the local dipolar field experienced by the nuclei, and $\xi$ is a numerical coefficient which depends on the nature of the spin-spin interactions [37]. The condition to induce spin precession by $\mathbf{B}_{\mathbf{n}}$ is given by $\mathbf{B}_{\mathbf{n}} \times \mathbf{S} \neq \mathbf{0}$. From Eq. (2), this condition is satisfied if $\mathbf{B}$ is applied along an oblique direction with respect to $\mathbf{S}$. Since $\mathbf{S}$ is parallel to the $x$-axis direction in the CMS/CoFe(1.1)/ $n$-GaAs sample, we applied $B$ along the oblique direction by $\theta=6^{\circ}$ from the $z$ axis in the $x-z$ plane-i.e., $\mathbf{B} \equiv B_{\mathrm{ob}}(\mathbf{x} \sin \theta+\mathbf{z} \cos \theta)$, where $B_{\mathrm{ob}}$ is the amplitude of the oblique field of $\mathbf{B}$ with both positive and negative signs, and $\mathbf{x}$ and $\mathbf{z}$ are unit vectors along the $x$-axis and $z$-axis directions, respectively. The total magnetic field of $\mathbf{B}+\mathbf{B}_{\mathbf{n}}$ induces Hanle precession for electron spins, resulting in a decrease of electron spin polarization under detector contact-3. Similarly to the conventional nonlocal Hanle signal, $V_{\mathrm{NL}}$ can be described by

$$
\begin{aligned}
V_{\mathrm{NL}}= & A \int_{0}^{\infty} \frac{1}{\sqrt{4 \pi D t}} \exp \left(-\frac{d^{2}}{4 D t}\right) \\
& \times \cos \left(\omega_{L} t\right) \exp \left(-\frac{t}{\tau_{s}}\right) d t,
\end{aligned}
$$

where $A$ is a constant, $D$ is the diffusion constant, $d$ is the distance between contact- 2 and contact- $3, \omega_{L}=g \mu_{\mathrm{B}} B_{z} / \hbar$ is the Larmor frequency, where $B_{z}$ is the $z$ component of $\mathbf{B}+\mathbf{B}_{\mathbf{n}}$, $g$ is an electron $g$ factor ( $g=-0.44$ for GaAs), $\mu_{\mathrm{B}}$ is the Bohr magneton, and $\hbar$ is the reduced Planck's constant. We ignored the influence of the $x$ or $y$ component of $\mathbf{B}+\mathbf{B}_{\mathbf{n}}$ on the Hanle signals because the direction of $\mathbf{B}+\mathbf{B}_{\mathbf{n}}$ was tilted only slightly (by $\theta=6^{\circ}$ ) from the $z$ axis. Figure 2(b) shows the $B_{\mathrm{ob}}$ dependence of $V_{\mathrm{NL}}$ for a CMS $/ \mathrm{CoFe}(1.1) / n$-GaAs sample in the parallel magnetization configuration between contact-2 and -3 with and without $\mathbf{B}_{\mathbf{a c}}$ at a frequency $(f)$ of $200 \mathrm{kHz}$. The device was initialized at $B_{\mathrm{ob}}=+48 \mathrm{mT}$ for a hold time ( $\left.t_{\text {hold }}\right)$ of $100 \mathrm{~s}$ at an injection current of $-70 \mu \mathrm{A}$, so that nuclear spins became dynamically polarized. The $P_{\mathrm{GaAs}}$, estimated from the amplitude of spin-valve signals, was approximately $12.8 \%$ at $I=-70 \mu \mathrm{A}$. The $B_{\mathrm{ob}}$ was then swept from $+48 \mathrm{mT}$ to $-48 \mathrm{mT}$ with a sweep rate of $0.17 \mathrm{mT} / \mathrm{s}$. This sweep rate was much faster than the time scale needed for the nuclear field to reach the steady state.

Under no irradiation of $\mathbf{B}_{\mathbf{a c}}$, we observed clear additional side peaks at $B_{\mathrm{ob}}=+24.3$ and $-6.8 \mathrm{mT}$. The behavior of $V_{\mathrm{NL}}$ as a function of $B_{\mathrm{ob}}$ is well described through Eqs. (1)-(3), and is explained in detail in Ref. [36]. We will focus here on the origin of the side peaks observed at $B_{\mathrm{ob}}=+24.3$ and $-6.8 \mathrm{mT}$. Since $b_{n}$ is negative, $\mathbf{B}_{\mathbf{n}}$ was generated along the direction antiparallel to $\mathbf{B}$ at $B_{\mathrm{ob}}=+48 \mathrm{mT}$. When $B_{\mathrm{ob}}$ reached $+24.3 \mathrm{mT}$ during the sweep, $\mathbf{B}_{\mathbf{n}}$ and $\mathbf{B}$ canceled 

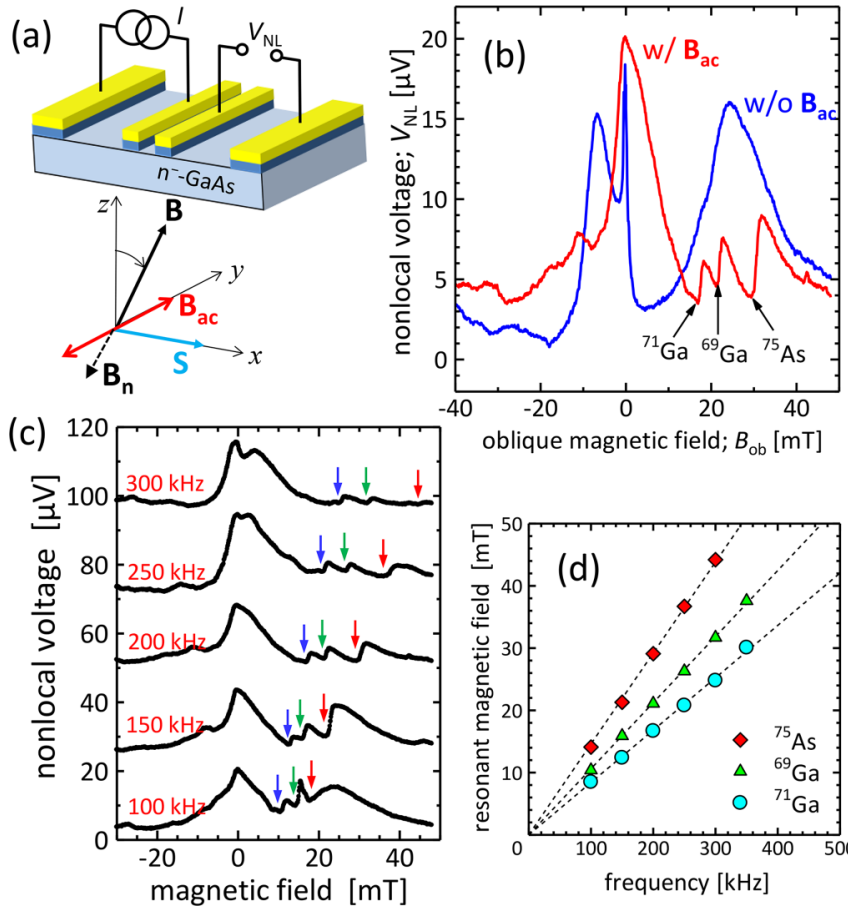

oblique magnetic field; $B_{\mathrm{ob}}[\mathrm{mT}]$

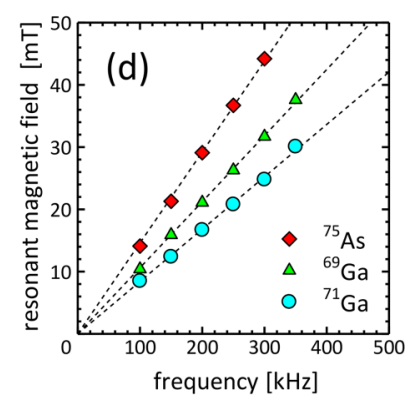

FIG. 2. (Color online) (a) Schematic device structure of a fourterminal lateral spin-transport device and circuit configuration for nonlocal measurements. $\mathbf{B}_{\mathbf{n}}$ was generated along an antiparallel direction to $\mathbf{B}$. (b) Transient oblique Hanle signals for a $\mathrm{Co}_{2} \mathrm{MnSi} / \mathrm{CoFe}(1.1) / n$-GaAs device with and without an rf magnetic field $\left(\mathbf{B}_{\mathrm{ac}}\right) . \mathbf{B}_{\mathrm{ac}}$ with its frequency of $200 \mathrm{kHz}$ was applied along the $y$-axis direction. (c) Transient oblique Hanle signals with frequencies of $\mathbf{B}_{\mathbf{a c}}$ ranging from 100 to $300 \mathrm{kHz}$. The signals are offset for clarity. (d) Frequency dependence of resonant magnetic field for ${ }^{75} \mathrm{As},{ }^{69} \mathrm{Ga}$, and ${ }^{71} \mathrm{Ga}$ nuclei.

each other, resulting in suppressed electron spin precession. Then, electron spins became polarized, causing $V_{\mathrm{NL}}$ to show a satellite peak at $B_{\mathrm{ob}}=+24.3 \mathrm{mT}$. Thus, one can evaluate the strength of $\mathbf{B}_{\mathbf{n}}$ through this measurement. The side peak at $B_{\mathrm{ob}}=-6.8 \mathrm{mT}$ was also caused by the cancellation of $\mathbf{B}$ and $\mathbf{B}_{\mathbf{n}}$. The asymmetric behavior of $V_{\mathrm{NL}}$ with respect to the polarity of $B_{\mathrm{ob}}$ is due to the transient response of the nuclear field to a reversal of $\mathbf{B}$, which is also explained in Ref. [36].

Under the irradiation of $\mathbf{c w}-\mathbf{B}_{\mathbf{a c}}$, the satellite peaks originating from the nuclear polarization decreased. In particular, a steep decrease of $V_{\mathrm{NL}}$ or dip structures indicated by arrows in Fig. 2(b) were observed at $B_{\mathrm{ob}}=30.6,22.0$, and $17.6 \mathrm{mT}$. These values are close to the resonant magnetic field of NMR for ${ }^{75} \mathrm{As},{ }^{69} \mathrm{Ga}$, and ${ }^{71} \mathrm{Ga}$, respectively. Thus, it is suggested that the change of $V_{\mathrm{NL}}$ by irradiation of $\mathbf{B}_{\mathrm{ac}}$ is due to the NMR effect; i.e., when $\mathbf{B}_{\mathbf{n}}$ decreased through the NMR, the total magnetic field experienced by electron spins increased, and the spin precession was enhanced, resulting in the decrease of $V_{\mathrm{NL}}$. To confirm this, we investigated the $f$ dependence of oblique Hanle signals. Figure 2(c) shows oblique Hanle signals for a CMS $/ \mathrm{CoFe}(1.1) / n$-GaAs sample with frequencies of $\mathbf{B}_{\mathbf{a c}}$ ranging from 100 to $300 \mathrm{kHz}$. As the $f$ increased, the dip positions shifted to a higher magnetic field. The values for these dips showed linear relations with $f$, as shown in Fig. 2(d). The slope for each line is in good agreement with that evaluated
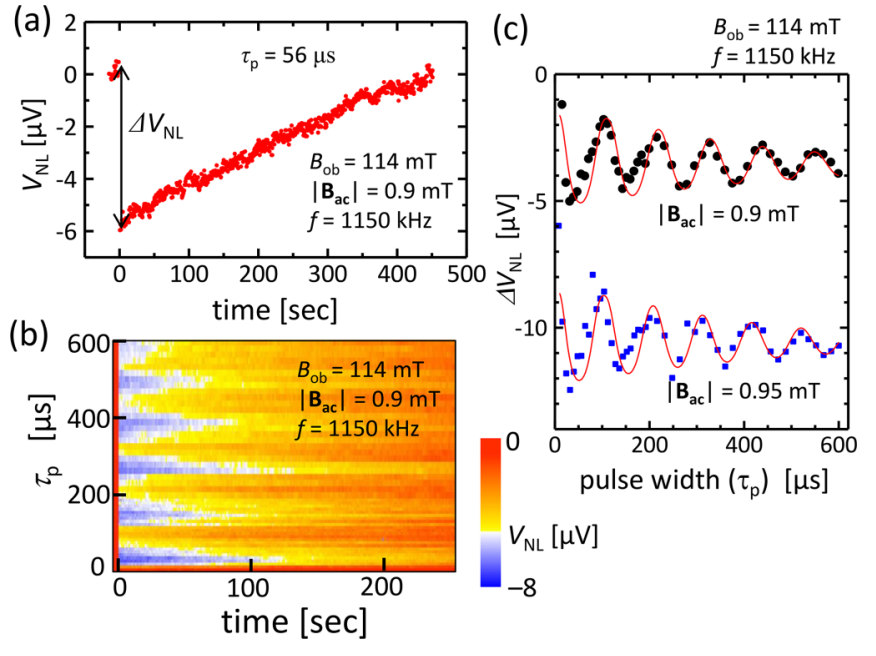

FIG. 3. (Color online) (a) Time $(t)$ evolution of nonlocal voltage $\left(V_{\mathrm{NL}}\right)$ for a $\mathrm{Co}_{2} \mathrm{MnSi} / \mathrm{CoFe}(1.3) / n$-GaAs device after irradiation of a pulsed $\mathbf{B}_{\mathbf{a c}}$ with a duration $\left(\tau_{p}\right)$ of $56 \mu$ s and frequency of $1150 \mathrm{kHz}$. The initial value of $V_{\mathrm{NL}}$ is set to zero. (b) A color-scale plot of $V_{\mathrm{NL}}$ as a function of $\tau_{p}$ and $t$. (c) Nonlocal voltage change vs rf-pulse duration (Rabi oscillation) for a $\mathrm{Co}_{2} \mathrm{MnSi} / \mathrm{CoFe}(1.3) / n$-GaAs device with different $\left|\mathbf{B}_{\mathbf{a c}}\right|$ values. The solid lines indicate approximated curves given by Eqs. (4) and (5).

from the gyromagnetic ratio of ${ }^{75} \mathrm{As},{ }^{69} \mathrm{Ga}$, and ${ }^{71} \mathrm{Ga}$ [37], respectively, within an error of $6 \%$. Thus, we conclude that the change of $V_{\mathrm{NL}}$ is originating from an NMR-induced decrease in nuclear polarization. Similar results were also obtained for a CMS $/ \mathrm{CoFe}(1.3) / n$-GaAs sample.

We will now describe a coherent rotation of nuclear spins using pulsed NMR. In the following we will focus on the NMR signal for ${ }^{69} \mathrm{Ga}$ in a CMS/CoFe $(1.3) / n$-GaAs device. We applied $\mathbf{B}$ along the oblique direction with $\theta \approx 5^{\circ}$ from the $z$ axis in the $x-z$ plane. The device was first initialized at $B_{\mathrm{ob}}=+114 \mathrm{mT}$ for $460 \mathrm{~s}$ at an injection current of $+90 \mu \mathrm{A}$, where electron spins were extracted from GaAs into CMS, resulting in $P_{\mathrm{GaAs}}$ being approximately $24.6 \%$. Since $\mathbf{B}$ and $\mathbf{B}_{\mathbf{n}}$ were almost canceled out by each other under this condition, electron spins were polarized, or no Hanle precession occurred under the initial condition. Figure 3(a) shows the time evolution of $V_{\mathrm{NL}}$ when an oscillatory magnetic field $\mathbf{B}_{\mathbf{a c}}$ with $f$ of $1150 \mathrm{kHz}$ and $\left|\mathbf{B}_{\mathbf{a c}}\right|$ of approximately $0.9 \mathrm{mT}$ was applied at $t=0$ for duration $\left(\tau_{p}\right)$ of $56 \mu \mathrm{s}$ (which we call a $\mathbf{B}_{\mathbf{a c}}$ hereafter), where $f$ of $1150 \mathrm{kHz}$ corresponded to the resonant frequency for ${ }^{69} \mathrm{Ga}$. Since the integrand of Eq. (3) can be ignored at $t>10 \tau_{s}$, one can evaluate the strength of $B_{z}$ through $V_{\mathrm{NL}}$ within a time scale of $10 \tau_{s}$ (typically several hundreds of nanoseconds). The $V_{\mathrm{NL}}$ decreased rapidly by $6.0 \mu \mathrm{V}$ right after the irradiation of the $\mathbf{B}_{\mathbf{a c}}$ pulse due to the NMR for ${ }^{69} \mathrm{Ga}$, and then it gradually recovered to its initial states with a time scale of several hundreds of seconds, which is comparable to those for DNP estimated from the $t_{\text {hold }}$ dependence of nuclear fields in our previous study [36]. The application of a $\mathbf{B}_{\mathbf{a c}}$ pulse with duration $\tau_{p}$ induces the rotation of nuclear spins by $\gamma\left({ }^{69} \mathrm{Ga}\right)\left|\mathbf{B}_{\mathbf{a c}}\right| \tau_{p}$ along the $y$ axis in the rotating coordinate system, which rotates along the $z$ axis by $\gamma\left({ }^{69} \mathrm{Ga}\right)|\mathbf{B}| \tau_{p}$, where $\gamma\left({ }^{69} \mathrm{Ga}\right)$ is the gyromagnetic ratio for 
${ }^{69} \mathrm{Ga}$. Thus, the $z$ component of the effective magnetic field, including the dephasing factor, is given by

$$
B_{z}\left(\tau_{p}\right)=A_{1}\left\{1-\cos \left(2 \pi f_{\text {Rabi }} \tau_{p}\right) \exp \left(-\tau_{p} / T_{2}^{\text {Rabi }}\right)\right\},
$$

where $A_{1}$ is a constant, $f_{\text {Rabi }}$ is the frequency for the oscillation of $B_{z}$ (or the Rabi frequency), which is equal to $\gamma\left({ }^{69} \mathrm{Ga}\right)\left|\mathbf{B}_{\mathbf{a c}}\right| / 2 \pi$, and $T_{2}{ }^{\text {Rabi }}$ is the effective dephasing time. We defined $\Delta V_{\mathrm{NL}}$ as an amount of change for $V_{\mathrm{NL}}$ just after the $\mathbf{B}_{\text {ac }}$ pulse. Substituting Eq. (4) into Eq. (3), we get

$$
\Delta V_{\mathrm{NL}}=\frac{A_{2}}{1+\left(g \mu_{\mathrm{B}} B_{z} \tau_{s}\right)^{2}}+A_{3},
$$

where $A_{2}$ and $A_{3}$ are constants. For simplicity we approximated Eq. (3) by the Lorentzian function. This approximation is valid for the case of $l_{\mathrm{sf}} \gg d$, a condition that is satisfied in our device. The oscillatory behavior of $B_{z}$ induces the oscillation of $\Delta V_{\mathrm{NL}}$ as a function of $\tau_{p}$. Figure 3(b) shows a color-scale plot of $V_{\mathrm{NL}}$ as a function of $\tau_{p}$ ranging from 8 to $600 \mu \mathrm{s}$ and $t$ from 0 to $250 \mathrm{~s}$, and Fig. 3(c) plots $\Delta V_{\mathrm{NL}}$ as a function of $\tau_{p}$ for different $\left|\mathbf{B}_{\mathbf{a c}}\right|$ values along with fitting curves calculated from Eq. (5). We observed clear oscillation of $\Delta V_{\mathrm{NL}}$ as a function of $\tau_{p}$ with $T_{2}{ }^{\text {Rabi }}$ of $\sim 400 \mu \mathrm{s}$, a value comparable to those reported in the literature ranging from 100 to $1500 \mu \mathrm{s}$ [8-12]. These results indicate the demonstration of Rabi oscillation in spin injection devices. Although there are some experimental errors for the first two periods, the plot of $\Delta V_{\mathrm{NL}}$ vs $\tau_{p}$ can be fitted well with Eqs. (4) and (5). The errors are possibly due to the distortion of the rf-pulse wave form for a certain number of initial periods or some of the approximations made to derive Eq. (5).

Note that we observed a sizable Rabi oscillation even at a relatively high temperature of $4.2 \mathrm{~K}$ and a low magnetic field of $\sim 0.1$ T compared with quantum Hall systems [8-10]. The ratio between the Zeeman splitting energy and the thermal energy, $\mu_{s(n)}|\mathbf{B}| / k_{\mathrm{B}} T$, where $\mu_{s(n)}$ is the magnetic moment of electron (nucleus), $k_{\mathrm{B}}$ is the Boltzmann constant, and $T$ is temperature, gives a measure of the electron (nuclear) spin polarization under thermal equilibrium. Even though both $\mu_{s}|\mathbf{B}| / k_{\mathrm{B}} T$ and $\mu_{n}|\mathbf{B}| / k_{\mathrm{B}} T$ were three orders of magnitude smaller in our devices than typical values in the quantum Hall system, spin injection from a half-metallic spin source enabled the creation of highly polarized electron spin states in GaAs, leading to a successful demonstration of coherent manipulation of nuclear spins. This is an advantage of our system from a device application point of view.

In conclusion, we have demonstrated coherent control of nuclear spins in bulk GaAs using spin injection from a half-metallic spin source of $\mathrm{Mn}$-rich $\mathrm{Co}_{2} \mathrm{MnSi}$. Efficient spin injection enabled efficient DNP and a sensitive detection of the NMR signal, leading to a sizable Rabi oscillation even at a low magnetic field. This approach provides a novel allelectrical solid-state NMR system with high spatial resolution and high sensitivity that can be used to implement scalable nuclear-spin-based qubits.

We thank Professor Kenji Kondo, Hokkaido University for his valuable discussion on the dynamics of nuclear spins. This work was partly supported by a Grant-in-Aid for Scientific Research (JSPS KAKENHI Grant No. 25286039) and by the Japan Science and Technology (JST) Agency through its Strategic International Cooperative Program under the title "Advanced spintronic materials and transport phenomena (ASPIMATT).” T.A. was also supported by a Research Fellowship for Young Scientists from the JSPS.
[1] L. M. K. Vandersypen, M. Steffen, G. Breyta, C. S. Yannoni, M. H. Sherwood, and I. L. Chuang, Nature (London) 414, 883 (2001).

[2] D. Gammon, S. W. Brown, E. S. Snow, T. A. Kennedy, D. S. Katzer, and D. Park, Science 277, 85 (1997).

[3] G. Salis, D. T. Fuchs, J. M. Kikkawa, D. D. Awschalom, Y. Ohno, and H. Ohno, Phys. Rev. Lett. 86, 2677 (2001).

[4] M. Poggio, G. M. Steeves, R. C. Myers, Y. Kato, A. C. Gossard, and D. D. Awschalom, Phys. Rev. Lett. 91, 207602 (2003).

[5] T. Machida, T. Yamazaki, and S. Komiyama, Appl. Phys. Lett. 80, 4178 (2002).

[6] K. Hashimoto, K. Muraki, T. Saku, and Y. Hirayama, Phys. Rev. Lett. 88, 176601 (2002).

[7] K. Ono and S. Tarucha, Phys. Rev. Lett. 92, 256803 (2004).

[8] T. Machida, T. Yamazaki, K. Ikushima, and S. Komiyama, Appl. Phys. Lett. 82, 409 (2003).

[9] G. Yusa, K. Muraki, K. Takashina, K. Hashimoto, and Y. Hirayama, Nature (London) 434, 1001 (2005).

[10] H. Takahashi, M. Kawamura, S. Masubuchi, K. Hamaya, T. Machida, Y. Hashimoto, and S. Katsumoto, Appl. Phys. Lett. 91, 092120 (2007).

[11] H. Sanada, Y. Kondo, S. Matsuzaka, K. Morita, C. Y. Hu, Y. Ohno, and H. Ohno, Phys. Rev. Lett. 96, 067602 (2006).
[12] Y. Kondo, M. Ono, S. Matsuzaka, K. Morita, H. Sanada, Y. Ohno, and H. Ohno, Phys. Rev. Lett. 101, 207601 (2008).

[13] X. Lou, C. Adelmann, S. A. Crooker, E. S. Garlid, J. Zhang, K. S. M. Reddy, S. D. Flexner, C. J. Palmstrøm, and P. A. Crowell, Nat. Phys. 3, 197 (2007).

[14] M. Ciorga, A. Einwanger, U. Wurstbauer, D. Schuh, W. Wegscheider, and D. Weiss, Phys. Rev. B 79, 165321 (2009).

[15] G. Salis, A. Fuhrer, R. R. Schlitter, L. Gross, and S. F. Alvarado, Phys. Rev. B 81, 205323 (2010).

[16] T. Uemura, T. Akiho, M. Harada, K.-i. Matsuda, and M. Yamamoto, Appl. Phys. Lett. 99, 082108 (2011).

[17] P. Bruski, Y. Manzke, R. Farshchi, O. Brandt, J. Herfort, and M. Ramsteiner, Appl. Phys. Lett. 103, 052406 (2013).

[18] T. Saito, N. Tezuka, M. Matsuura, and S. Sugimoto, Appl. Phys. Express 6, 103006 (2013).

[19] T. Sasaki, T. Oikawa, T. Suzuki, M. Shiraishi, Y. Suzuki, and K. Noguchi, IEEE Trans. Magn. 46, 1436 (2010).

[20] Y. Saito, M. Ishikawa, T. Inokuchi, H. Sugiyama, T. Tanamoto, K. Hamaya, and N. Tezuka, IEEE Trans. Magn. 48, 2739 (2012).

[21] T. Sasaki, Y. Ando, M. Kameno, T. Tahara, H. Koike, T. Oikawa, T. Suzuki, and M. Shiraishi, Phys. Rev. Appl. 2, 034005 (2014).

[22] Y. Zhou, W. Han, L.-T. Chang, F. Xiu, M. Wang, M. Oehme, I. A. Fischer, J. Schulze, R. K. Kawakami, and K. L. Wang, Phys. Rev. B 84, 125323 (2011). 
[23] P. V. Dorpe, W. V. Roy, J. D. Boeck, and G. Borghs, Phys. Rev. B 72, 035315 (2005).

[24] J. Strand, X. Lou, C. Adelmann, B. D. Schultz, A. F. Isakovic, C. J. Palmstrøm, and P. A. Crowell, Phys. Rev. B 72, 155308 (2005).

[25] M. K. Chan, Q. O. Hu, J. Zhang, T. Kondo, C. J. Palmstrøm, and P. A. Crowell, Phys. Rev. B 80, 161206(R) (2009).

[26] J. Shiogai, M. Ciorga, M. Utz, D. Schuh, T. Arakawa, M. Kohda, K. Kobayashi, T. Ono, W. Wegscheider, D. Weiss, and J. Nitta, Appl. Phys. Lett. 101, 212402 (2012).

[27] H.-x. Liu, Y. Honda, T. Taira, K.-i. Matsuda, M. Arita, T. Uemura, and M. Yamamoto, Appl. Phys. Lett. 101, 132418 (2012).

[28] H.-x. Liu, T. Kawami, K. Moges, T. Uemura, M. Yamamoto, F. Shi, and P. M. Voyles, J. Phys. D: Appl. Phys. 48, 164001 (2015).

[29] M. Yamamoto, T. Ishikawa, T. Taira, G.-f. Li, K.-i. Matsuda, and T. Uemura, J. Phys.: Condens. Matter 22, 164212 (2010).

[30] G.-f. Li, Y. Honda, H.-x. Liu, K.-i. Matsuda, M. Arita, T. Uemura, M. Yamamoto, Y. Miura, M. Shirai, T. Saito, F. Shi, and P. M. Voyles, Phys. Rev. B 89, 014428 (2014).
[31] J.-P. Wüstenberg, R. Fetzer, M. Aeschlimann, M. Cinchetti, J. Minár, Jürgen Braun, H. Ebert, T. Ishikawa, T. Uemura, and M. Yamamoto, Phys. Rev. B 85, 064407 (2012).

[32] V. R. Singh, V. K. Verma, K. Ishigami, G. Shibata, T. Kadono, A. Fujimori, D. Asakura, T. Koide, Y. Miura, M. Shirai, G.-f. Li, T. Taira, and M. Yamamoto, Phys. Rev. B 86, 144412 (2012).

[33] Y. Ebina, T. Akiho, H.-x. Liu, M. Yamamoto, and T. Uemura, Appl. Phys. Lett. 104, 172405 (2014).

[34] X. Y. Dong, C. Adelmann, J. Q. Xie, C. J. Palmstrøm, X. Lou, J. Strand, P. A. Crowell, J.-P. Barnes, and A. K. Petford-Long, Appl. Phys. Lett. 86, 102107 (2005).

[35] M. Ramsteiner, O. Brandt, T. Flissikowski, H. T. Grahn, M. Hashimoto, J. Herfort, and H. Kostial, Phys. Rev. B 78, 121303(R) (2008).

[36] T. Akiho, J. Shan, H.-x. Liu, K.-i. Matsuda, M. Yamamoto, and T. Uemura, Phys. Rev. B 87, 235205 (2013).

[37] D. Paget and V. L. Berkovits, in Optical Orientation, edited by F. Meier and B. P. Zakharchenya (North-Holland, New York, 1984). 